\title{
Toxicity evaluation of standardized and nanoliposomal extracts of Labisia pumila whole plant (Blume, Myrsinaceae) in Sprague Dawley rats
}

\author{
Mohammed Ali Ahmed Saeed ${ }^{1}$, Abdul Hakeem Memon ${ }^{2}$, Mohd Shahrul Ridzuan \\ Hamil $^{1}$, Hooi Kheng Beh ${ }^{3}$, Sultan Ayesh Mohammed Saghir ${ }^{4}$, Gurjeet Kaur ${ }^{5}$, \\ Amirin Sadikun ${ }^{1}$, Zhari Ismail ${ }^{1 *}$ \\ ${ }^{1}$ Department of Pharmaceutical Chemistry, School of Pharmaceutical Sciences, Universiti Sains Malaysia, ${ }^{2}$ Department of \\ Pharmacognosy, Faculty of Pharmacy, University of Sindh, Jamashoro, Pakistan, ${ }^{3}$ Faculty of Agro Based Industry, Universiti \\ Malaysia Kelantan, 16100 Kota Bharu, Kelantan, Malaysia, ${ }^{4}$ Department of Pharmacology, School of Pharmaceutical Sciences, \\ ${ }^{5}$ Institute for Research in Molecular Medicine, Universiti Sains Malaysia, 11800 Minden, Pulau Pinang, Malaysia
}

*For correspondence: Email: ismailzhari@gmail.com; Tel: 006-046532242

\begin{abstract}
Purpose: To investigate the toxicity of Labisia pumila standardized extract (LPE) and its liposomal extract (LLP).

Methods: For acute toxicity study, LPE or LLP was orally administered $(2000 \mathrm{mg} / \mathrm{kg}$ ) in single doses to Sprague Dawley rats and the routine activity of the rats was continuously monitored for a total of 14 days. After 14 days of treatment, all rats were sacrificed and their vital organs were excised, weighed and macroscopically examined, while for a repeated dose toxicity study, the rats were orally administered with LPE or LLP at the selected doses $(250,500$ and $1000 \mathrm{mg} / \mathrm{kg})$ for a period of 28 days. The animals were sacrificed (anaesthetized by sodium pentobarbitone and blood was collected by cardiac puncture), followed by examination of their body organs and blood serum.

Results: LPE and LLP at $2000 \mathrm{mg} / \mathrm{kg}$ did not produce mortality or significant changes in the general behaviour, body weight and organ gross appearance of the rats. In repeated dose toxicity study no significant changes in, growth, organ weights, haematological parameters, biochemical values and histological features of vital organs of the treated groups, compared to the control group.

Conclusion: The no-adverse-effect-level for LPE and LLP is (1000 mg/kg/day) when administered orally for 28 days.
\end{abstract}

Keywords: Labisia pumila, Acute oral toxicity, Repeated dose toxicity

\begin{abstract}
This is an Open Access article that uses a funding model which does not charge readers or their institutions for access and distributed under the terms of the Creative Commons Attribution License (http://creativecommons.org/licenses/by/4.0) and the Budapest Open Access Initiative (http://www.budapestopenaccessinitiative.org/read), which permit unrestricted use, distribution, and reproduction in any medium, provided the original work is properly credited.
\end{abstract}

Tropical Journal of Pharmaceutical Research is indexed by Science Citation Index (SciSearch), Scopus, International Pharmaceutical Abstract, Chemical Abstracts, Embase, Index Copernicus, EBSCO, African Index Medicus, JournalSeek, Journal Citation Reports/Science Edition, Directory of Open Access Journals (DOAJ), African Journal Online, Bioline International, Open-J-Gate and Pharmacy Abstracts

\section{INTRODUCTION}

Labisia pumila Blume, var. alata (Family: Myrsinaceae), known as Kacip Fatimah in Malaysia. Traditionally, L. pumila has been used due to its medicinal values in health products and female tonics. Furthermore, L. pumila is used in other ailments such as dysmenorrhoea, gonorrhoea, dysentery and flatulence [1]. Recent pharmacological studies have reported that $L$. 
pumila has phytoestrogenic, anti-agiogenesis, anticancer, antioxidant, antibacterial, antifungal, anti-aging and anti-inflammatoryactivities. The benefits of the traditional use of $L$. pumila have also been supported by identification and isolation of many active compounds including phenolics, flavonoids and resorcinol compounds [2]. Gallic acid (as one of the isolated compounds from this plant) possessed many pharmacological activities such as antioxidant, antiangiogenesis, antiobesity, and anticancer effects. Rutin is also reported for different pharmacological effects, for example hepatic protective, antidiabetic, antihypertensive, antiinflammatory, anticancer and antioxidant activities. Gallic acid and rutin both found in $L$. pumila extract [3-8]. Sub-acute, teratogenic and reproductive toxicity studies of $L$. pumila water extract at $(50,1000$ and $800 \mathrm{mg} / \mathrm{kg})$, respectively without any side effects were reported [9-11]. The present study has been designed to perform the acute oral toxicity study at $(2000 \mathrm{mg} / \mathrm{kg})$ and repeated dose oral toxicity study of LPE and LLP at $(250,500$ and $1000 \mathrm{mg} / \mathrm{kg})$, respectively. Furthermore, the present study also describe authenticity of plant species, parts of plant, type of extract and method of extraction. So, this study was aimed to evaluate the acute and repeated dose oral toxicity studies on standardized L. pumila extract (LPE) and liposome of L. pumila (LLP).

\section{EXPERIMENTAL}

\section{Chemical and reagents}

Soybean lecithin was procured from Hong Aun kimia Sdn Bhd., Malaysia. Acetone, chloroform and ethanol were obtained from Quality Reagent Chemical Malaysia.

\section{Preparation of LPE and LLP}

The whole plant of $L$. pumila var. alata was extracted with ethanol $(50 \%)$ using soxhlet apparatus. The crude extract was filtered, concentrated under reduced pressure using a rotary evaporator, and further dried in oven. The dried extract was then kept in the fridge at $-20^{\circ} \mathrm{C}$ prior to use. Liposome formulation of ethanol $(50 \%)$ extract of L. pumila was prepared as described by [12].

\section{Acute oral toxicity study}

Male and female Sprague Dawley rats at 8-10 weeks of age were used. The animals were acclimatized to laboratory conditions for a week prior to the experiment. The rats were housed with free access to feed (normal laboratory chow,
Gold Coin) and tap water ad libitum. The rats were maintained at $28 \pm 2^{\circ} \mathrm{C}$ under a light/dark cycle of $12 \mathrm{~h}$. All the procedures were done according to the OECD guidelines (2008) [13 and 15] and approved by the Animal Ethics Committee of Universiti Sains Malaysia (USM/Animal Ethics approval no. 2014/597).

LPE and LLP were administered orally (2000 $\mathrm{mg} / \mathrm{kg})$ in single doses to the rats $(n=5)$. Another group of 5 rats without treatment was used as control group. The general behaviour of the rats were continuously monitored for $1 \mathrm{~h}$ after dosing, periodically during the first $24 \mathrm{~h}$ and daily thereafter for a total of 14 days. After 14 days of treatment, all animals were euthanized in $\mathrm{CO}_{2}$ chamber, and selected vital organs were excised, weighed, and macroscopically examined [14].

\section{Repeated dose toxicity study}

Animals (70 rats) were divided into 7 groups (5 rats/sex/group) and received the test materials orally. Group 1 served as control receiving vehicle only. Animals in group 2 to group 7 received 250,500 and $1000 \mathrm{mg} / \mathrm{kg}$ of LPE or LLP, respectively. Animals were treated once daily consecutively for 28 days. The animals were observed daily for clinical signs and mortality. At the end of the experiment, animals were anaesthetized by sodium pentobarbitone and blood was collected by cardiac puncture from overnight fasted animals. Vital organs such as brain, heart, lungs, liver, spleen, kidneys, and testes/ovaries were removed and weighed. Haematological, biochemical and histological analysis were done [15]. Measurements were made by (Gribbles Lab., Penang, Malaysia).

\section{Statistical analysis}

Data are expressed as mean \pm standard error of mean (SEM) and were analysed using Statistical Package for the Social Sciences (SPSS 20.0, IBM). The data were considered significant at $p<$ 0.05 .

\section{RESULTS}

\section{Toxicity of LPE and LLP}

For acute oral toxicity study, lethal effects were not observed after administration of LPE or LLP at dose $(2000 \mathrm{mg} / \mathrm{kg})$. No behavioural changes were observed during the observation period. There were no significant changes in the organto-body weight ratios (Table 1) of the treated rats compared to normal control group. Therefore, the 
$\mathrm{LD}_{50}$ value for oral administration of LPE and LLP was greater than $2000 \mathrm{mg} / \mathrm{kg}$.

In repeated dose oral toxicity study, there was no mortality of animals at any dose. Gross observations did not show any changes and all animals of both groups remain healthy. There were no abnormalities founded with respect to eye colour, touch response, salivation, grip strength and tail pinch. No locomotor dysfunction, convulsions or tremors were observed in all animals.

There were no significant differences observed for the weekly body weight and organ-to-body weight ratios of rats treated with LPE or LLP for 28 days (Table 2 and Table 3 ) except for LLP $250 \mathrm{mg} / \mathrm{kg}$, LPE $500 \mathrm{mg} / \mathrm{kg}$, and LLP $500 \mathrm{mg} / \mathrm{kg}$ for female groups during 14 days as presented in Table 2. The haematology results (Table 4) showed no statistically significant differences in the values for treated groups compared to normal control group except for haemoglobin $(\mathrm{Hb})$, packed cell volume (PCV), mean cell volume $(\mathrm{MCV})$, and neutrophiles in female groups only but still within the normal range as previously reported [16]. A biochemical examination results are summarized in Table 5, the levels of biochemical parameters were not statistically significant compared to normal control groups except for urea and uric acid in female treated groups, and for total cholesterol (TC), triglycerides (TG), and high density lipoprotein (HDL) in female and male treated groups but remained within the normal range except for urea level in female treated groups (LPE $500 \mathrm{mg} / \mathrm{kg}$ and LLP at 250, 500, 1000 $\mathrm{mg} / \mathrm{kg}$ ). Among the indicators of liver function, alanine aminotransferase (ALT), Aspartate aminotransferase (AST) and alkaline phosphatase (ALP) were not affected by LPE or LLP in both male and female treated groups.

The histological examination of liver and kidney tissues of normal control group and treated groups did not show any pathological abnormalities. Both control and treated groups showed normal hepatic architecture. Mild periportal inflammation was seen in female and male normal control groups (only 1 out of 5 rats), female LPE $500 \mathrm{mg} / \mathrm{kg}$ group (only 1 out of 5 rats) and male LPE 250 and $1000 \mathrm{mg} / \mathrm{kg}$ groups (only 1 out of 5 rats), while it didn't show any periportal chronic inflammation in liver of LLP treated groups in both male and female rats.

Table 1: Organ-to-body weight ratios of rats treated with LPE and LLP for acute toxicity study

\begin{tabular}{llll}
\hline Organ & $\begin{array}{l}\text { Normal control } \\
\text { water }(\mathbf{1 0} \mathbf{~} \mathbf{L} / \mathbf{k g})\end{array}$ & $\begin{array}{l}\text { Treated group with LPE } \\
\mathbf{( 2 0 0 0} \mathbf{~} \mathbf{g} / \mathbf{k g})\end{array}$ & $\begin{array}{l}\text { Treated group with LLP } \\
\mathbf{( 2 0 0 0} \mathbf{~} \mathbf{g} / \mathbf{k g})\end{array}$ \\
\hline Heart & $0.35 \pm 0.01$ & $0.35 \pm 0.01$ & $0.32 \pm 0.01$ \\
Liver & $3.12 \pm 0.09$ & $2.85 \pm 0.08$ & $3.11 \pm 0.09$ \\
kidney (R) & $0.32 \pm 0.01$ & $0.33 \pm 0.01$ & $0.31 \pm 0.01$ \\
kidney (L) & $0.32 \pm 0.01$ & $0.32 \pm 0.01$ & $0.32 \pm 0.01$ \\
Lung & $0.63 \pm 0.04$ & $0.62 \pm 0.04$ & $0.64 \pm 0.02$ \\
adrenal (R) & $0.02 \pm 0.00$ & $0.02 \pm 0.00$ & $0.02 \pm 0.00$ \\
adrenal (L) & $0.02 \pm 0.00$ & $0.02 \pm 0.00$ & $0.02 \pm 0.00$ \\
Spleen & $0.26 \pm 0.01$ & $0.28 \pm 0.01$ & $0.27 \pm 0.02$ \\
Uterus & $0.22 \pm 0.04$ & $0.26 \pm 0.06$ & $0.23 \pm 0.01$ \\
Ovaries & $0.18 \pm 0.03$ & $0.19 \pm 0.02$ & $0.19 \pm 0.01$ \\
Thymus & $0.19 \pm 0.01$ & $0.21 \pm 0.02$ & $0.18 \pm 0.01$ \\
Brain & $0.75 \pm 0.03$ & $0.72 \pm 0.01$ & $0.74 \pm 0.01$ \\
\hline
\end{tabular}

Values are expressed as mean \pm SEM. No significant difference between normal control group vs. treated groups, where $R=$ right and $L=$ left

Table 2: Weekly body weight $(\mathrm{g})$ of rats treated with LPE and LLP for 28 days

\begin{tabular}{lccccc}
\hline Group & 0 day & 7 days & 14 days & 21 days & 28 days \\
\hline Normal control & $218.15 \pm 8.16$ & $233.5 \pm 9.47$ & $249.1 \pm 11.10$ & $233.4 \pm 9.95$ & $239.66 \pm 11.07$ \\
LPE $250 \mathrm{mg} / \mathrm{kg}$ & $217.96 \pm 7.38$ & $226.77 \pm 6.73$ & $233.9 \pm 8.85$ & $224.5 \pm 9.57$ & $235.74 \pm 9.27$ \\
LPE $500 \mathrm{mg} / \mathrm{kg}$ & $219.1 \pm 4.78$ & $223.85 \pm 3.91$ & $232.4 \pm 7.05$ & $220.1 \pm 5.50$ & $235.69 \pm 6.53$ \\
LPE $1000 \mathrm{mg} / \mathrm{kg}$ & $209.44 \pm 3.81$ & $218.49 \pm 6.99$ & $230.6 \pm 7.35$ & $218 \pm 7.48$ & $227.51 \pm 8.97$ \\
LLP $250 \mathrm{mg} / \mathrm{kg}$ & $217.49 \pm 7.63$ & $226.84 \pm 5.82$ & $226.53 \pm 6.16$ & $235.94 \pm 7.02$ & $228.24 \pm 6.82$ \\
LLP $500 \mathrm{mg} / \mathrm{kg}$ & $217.86 \pm 5.34$ & $232.66 \pm 6.47$ & $233.97 \pm 7.30$ & $243.06 \pm 7.54$ & $234.77 \pm 8.90$ \\
LLP $1000 \mathrm{mg} / \mathrm{kg}$ & $230.81 \pm 5.28$ & $229.54 \pm 6.41$ & $227.32 \pm 6.02$ & $235.21 \pm 5.85$ & $224.91 \pm 8.04$ \\
\hline Values are expressed as mean \pm SEM. $(\mathrm{n}=10) ;{ }^{*}=p<0.05,{ }^{* *}=p<0.01,{ }^{\star \star *}=p<0.001$ for treated groups vs. \\
normal control
\end{tabular}


Table 3: Organ-to-body weight ratios of rats treated with LPE and LLP for 28 days

\begin{tabular}{|c|c|c|c|c|c|c|c|}
\hline $\begin{array}{l}\text { \% Organ weight/ } \\
\text { Bodyweight }\end{array}$ & Normal control & $\begin{array}{c}\text { LPE } \\
250 \mathrm{mg} / \mathrm{kg}\end{array}$ & $\begin{array}{c}\text { LPE } \\
500 \mathrm{mg} / \mathrm{kg}\end{array}$ & $\begin{array}{c}\text { LPE } \\
1000 \mathrm{mg} / \mathrm{kg}\end{array}$ & $\begin{array}{c}\text { LLP } \\
250 \mathrm{mg} / \mathrm{kg}\end{array}$ & $\begin{array}{c}\text { LLP } \\
500 \mathrm{mg} / \mathrm{kg}\end{array}$ & $\begin{array}{c}\text { LLP } \\
1000 \mathrm{mg} / \mathrm{kg}\end{array}$ \\
\hline Brain & $0.74 \pm 0.025$ & $0.75 \pm 0.025$ & $0.75 \pm 0.16$ & $0.75 \pm 0.035$ & $0.74 \pm 0.02$ & $0.73 \pm 0.025$ & $0.72 \pm 0.05$ \\
\hline Heart & $0.34 \pm 0.015$ & $0.35 \pm 0.015$ & $0.36 \pm 0.01$ & $0.35 \pm 0.015$ & $0.33 \pm 0.01$ & $0.34 \pm 0.025$ & $0.32 \pm 0.015$ \\
\hline Liver & $2.68 \pm 0.075$ & $2.61 \pm 0.085$ & $2.65 \pm 0.09$ & $2.61 \pm 0.095$ & $2.74 \pm 0.1$ & $2.73 \pm 0.17$ & $2.52 \pm 0.105$ \\
\hline Spleen & $0.21 \pm 0.01$ & $0.22 \pm 0.015$ & $0.21 \pm 0.01$ & $0.21 \pm 0.015$ & $0.20 \pm 0.01$ & $0.20 \pm 0.01$ & $0.19 \pm 0.01$ \\
\hline Kidney (L) & $0.32 \pm 0.015$ & $0.32 \pm 0.01$ & $0.30 \pm 0.02$ & $0.32 \pm 0.015$ & $0.30 \pm 0.015$ & $0.30 \pm 0.015$ & $0.29 \pm 0.01$ \\
\hline Kidney (R) & $0.32 \pm 0.015$ & $0.33 \pm 0.015$ & $0.32 \pm 0.015$ & $0.32 \pm 0.01$ & $0.31 \pm 0.015$ & $0.30 \pm 0.015$ & $0.30 \pm 0.015$ \\
\hline Thymus & $0.20 \pm 0.02$ & $0.17 \pm 0.025$ & $0.19 \pm 0.025$ & $0.21 \pm 0.035$ & $0.19 \pm 0.025$ & $0.19 \pm 0.025$ & $0.19 \pm 0.03$ \\
\hline
\end{tabular}

Values are expressed as mean \pm SEM. $(n=10)$. No statistical significant difference for treated groups vs. normal control.

Table 4: Haematological values of rats treated with LPE and LLP for 28 days

\begin{tabular}{|c|c|c|c|c|c|c|c|}
\hline $\begin{array}{c}\text { Hematological } \\
\text { parameters }\end{array}$ & Normal control & $\begin{array}{c}\text { LPE } \\
250 \mathrm{mg} / \mathrm{kg}\end{array}$ & $\begin{array}{c}\text { LPE } \\
500 \mathrm{mg} / \mathrm{kg}\end{array}$ & $\begin{array}{c}\text { LPE } \\
1000 \mathrm{mg} / \mathrm{kg}\end{array}$ & $\begin{array}{c}\text { LLP } \\
250 \mathrm{mg} / \mathrm{kg}\end{array}$ & $\begin{array}{c}\text { LLP } \\
500 \mathrm{mg} / \mathrm{kg}\end{array}$ & $\begin{array}{c}\text { LLP } \\
1000 \mathrm{mg} / \mathrm{kg}\end{array}$ \\
\hline $\mathrm{Hb}(\mathrm{g} / \mathrm{dL})$ & $169.3 \pm 2.58$ & $167.20 \pm 2.49$ & $162.60 \pm 13.91$ & $170.10 \pm 4.23$ & $154.60 \pm 2.36$ & $160.30 \pm 3.40$ & $154.90 \pm 1.19$ \\
\hline $\operatorname{RBC}\left(\times 10^{12} / \mathrm{L}\right)$ & $9.23 \pm 0.18$ & $9.18 \pm 0.14$ & $8.17 \pm 0.93$ & $9.04 \pm 0.20$ & $8.25 \pm 0.16$ & $8.71 \pm 0.14$ & $8.46 \pm 0.13$ \\
\hline $\mathrm{PCV}(\mathrm{L} / \mathrm{L})$ & $0.52 \pm 0.01$ & $0.53 \pm 0.01$ & $0.49 \pm 0.06$ & $0.53 \pm 0.02$ & $0.48 \pm 0.02$ & $0.49 \pm 0.01$ & $0.46 \pm 0.01^{*}$ \\
\hline MCV (gl) & $56.90 \pm 0.97$ & $58.20 \pm 0.91$ & $59.80 \pm 1.52$ & $58.50 \pm 1.38$ & $58.30 \pm 1.31$ & $55.50 \pm 1.03$ & $54.30 \pm 0.44$ \\
\hline White cell count $\left(\times 10^{y} / \mathrm{L}\right)$ & $5.32 \pm 0.96$ & $4.44 \pm 0.40$ & $4.69 \pm 0.96$ & $6.32 \pm 1.06$ & $4.08 \pm 0.68$ & $3.70 \pm 0.63$ & $3.50 \pm 0.84$ \\
\hline Neutrophil $\left(\times 10^{y} / \mathrm{L}\right)$ & $1.73 \pm 0.38$ & $1.63 \pm 0.17$ & $1.89 \pm 0.59$ & $1.83 \pm 0.38$ & $1.19 \pm 0.19$ & $0.83 \pm 0.07^{* \star *}$ & $0.76 \pm 0.17^{\star \star *}$ \\
\hline Lymphocytes $\left(\times 10^{y} / \mathrm{L}\right)$ & $3.35 \pm 0.66$ & $2.65 \pm 0.33$ & $2.57 \pm 0.48$ & $4.15 \pm 0.66$ & $2.68 \pm 0.48$ & $2.68 \pm 0.51$ & $2.53 \pm 0.74$ \\
\hline Monocytes $\left(\times 10^{y} / \mathrm{L}\right)$ & $0.16 \pm 0.05$ & $0.11 \pm 0.03$ & $0.14 \pm 0.07$ & $0.17 \pm 0.05$ & $0.13 \pm 0.03$ & $0.17 \pm 0.04$ & $0.14 \pm 0.27$ \\
\hline Eosinophils $\left(\times 10^{y} / L\right)$ & $0.10 \pm 0.02$ & $0.12 \pm 0.02$ & $0.16 \pm 0.07$ & $0.115 \pm 0.03$ & $0.13 \pm 0.03$ & $0.13 \pm 0.02$ & $0.12 \pm 0.01$ \\
\hline Platelets $\left(\times 10^{y} / \mathrm{L}\right)$ & $1065.10 \pm 87.45$ & $778 \pm 112.16$ & $676.60 \pm 155.56$ & $604 \pm 77.98$ & $726.90 \pm 97.43$ & $962.40 \pm 60.66$ & $874.50 \pm 50.37$ \\
\hline
\end{tabular}

Values are expressed as mean \pm SEM. $(\mathrm{n}=10) ;{ }^{*}=p<0.05,{ }^{* *}=p<0.01,{ }^{* * *}=p<0.001$ for treated groups vs. normal control. Hb $=$ haemoglobin, RBC $=$ red blood cells, PCV $=$ packed cell volume and $\mathrm{MCV}=$ mean cell volume. 
Table 5: Biochemical parameters of rats treated with LPE and LLP for 28 days

\begin{tabular}{|c|c|c|c|c|c|c|c|}
\hline $\begin{array}{l}\text { Biochemical } \\
\text { parameters }\end{array}$ & $\begin{array}{l}\text { Normal } \\
\text { control }\end{array}$ & $\begin{array}{c}\text { LPE } \\
250 \mathrm{mg} / \mathrm{kg}\end{array}$ & $\begin{array}{c}\text { LPE } \\
500 \mathrm{mg} / \mathrm{kg}\end{array}$ & $\begin{array}{c}\text { LPE } \\
1000 \mathrm{mg} / \mathrm{kg}\end{array}$ & $\begin{array}{c}\text { LLP } \\
250 \mathrm{mg} / \mathrm{kg}\end{array}$ & $\begin{array}{c}\text { LLP } \\
500 \mathrm{mg} / \mathrm{kg}\end{array}$ & $\begin{array}{c}\text { LLP } \\
1000 \mathrm{mg} / \mathrm{kg}\end{array}$ \\
\hline Urea (mmol/L) & $8.00 \pm 0.27$ & $8.00 \pm 0.37$ & $8.69 \pm 0.34$ & $8.06 \pm 0.36$ & $6.96 \pm 0.31^{*}$ & $6.73 \pm 0.34^{\star *}$ & $6.06 \pm 0.34^{\star \star}$ \\
\hline Creatinine (umol/L) & $49.20 \pm 2.14$ & $54.20 \pm 2.19$ & $54.10 \pm 2.47$ & $47.90 \pm 3.51$ & $45.20 \pm 2.14$ & $43.00 \pm 1.95$ & $41.10 \pm 3.48$ \\
\hline Uric acid (mmol/L) & $0.13 \pm 0.01$ & $0.14 \pm 0.01$ & $0.13 \pm 0.01$ & $0.12 \pm 0.01$ & $0.15 \pm 0.03$ & $0.13 \pm 0.02$ & $0.13 \pm 0.02$ \\
\hline $\mathrm{TP}(\mathrm{g} / \mathrm{L})$ & $80.60 \pm 1.44$ & $82.20 \pm 1.61$ & $80.60 \pm 0.82$ & $79.30 \pm 1.65$ & $76.50 \pm 1.59$ & $77.40 \pm 1.25$ & $74.50 \pm 1.56$ \\
\hline ALP (U/L) & $117.10 \pm 13.67$ & $118.10 \pm 15.57$ & $138.90 \pm 22.40$ & $136.00 \pm 22.31$ & $136.70 \pm 14.73$ & $130.00 \pm 22.47$ & $111.70 \pm 12.90$ \\
\hline AST (U/L) & $274.20 \pm 12.59$ & $299.20 \pm 17.50$ & $272.90 \pm 16.60$ & $310.80 \pm 20.74$ & $233.60 \pm 20.84$ & $244.90 \pm 24.20$ & $227.90 \pm 24.87$ \\
\hline ALT (U/L) & $50.80 \pm 3.17$ & $56.00 \pm 3.08$ & $54.40 \pm 4.55$ & $50.50 \pm 3.14$ & $59.20 \pm 9.17$ & $53.80 \pm 10.52$ & $43.80 \pm 4.74$ \\
\hline Glu. (mmol/L) & $3.78 \pm 0.51$ & $3.83 \pm 0.29$ & $4.21 \pm 0.28$ & $4.04 \pm 0.38$ & $5.57 \pm 0.70$ & $5.33 \pm 0.86$ & $5.67 \pm 0.27$ \\
\hline TC (mmol/L) & $3.02 \pm 0.18$ & $3.30 \pm 0.27$ & $3.13 \pm 0.16$ & $3.02 \pm 0.23$ & $1.68 \pm 0.10$ & $1.80 \pm 0.16$ & $1.68 \pm 0.13$ \\
\hline $\mathrm{TG}(\mathrm{mmol} / \mathrm{L})$ & $0.35 \pm 0.02$ & $0.45 \pm 0.03^{*}$ & $0.43 \pm 0.02$ & $0.42 \pm 0.03$ & $0.71 \pm 0.06^{* * *}$ & $0.73 \pm 0.12^{\star *}$ & $0.54 \pm 0.07^{\star *}$ \\
\hline LDL (mmol/L) & $1.09 \pm 0.21$ & $1.41 \pm 0.25$ & $1.15 \pm 0.19$ & $1.13 \pm 0.43$ & $0.95 \pm 0.07$ & $1.02 \pm 0.13$ & $0.98 \pm 0.12$ \\
\hline $\mathrm{HDL}(\mathrm{mmol} / \mathrm{L})$ & $1.77 \pm 0.17$ & $1.69 \pm 0.17$ & $1.82 \pm 0.12$ & $1.70 \pm 0.22$ & $0.41 \pm 0.03^{* * *}$ & $0.45 \pm 0.04^{* * *}$ & $0.46 \pm 0.05^{\star * *}$ \\
\hline
\end{tabular}

Values are expressed as mean \pm SEM. $(n=10) ; ;^{*} p<0.05,{ }^{\star *} p<0.01,{ }^{\star \star *} p<0.001$ for treated groups vs. normal control. TP $=$ total protein, ALP $=$ alkaline phosphate, AST $=$

Aspartate aminotransferase, $\mathrm{ALT}=$ alanine aminotransferase, $\mathrm{Glu}=$ glucose, $\mathrm{TC}=$ cholesterol, $\mathrm{TG}=$ triglycerides, $\mathrm{LDL}=$ low density lipoprotein and $\mathrm{HDL}=$ high density lipoprotein. 
No ballooning degeneration, inflammation or other pathological abnormalities were observed in liver tissue samples of male and female rats (Plates 1 and 2). Kidney exhibited normal renal cortex containing normal glomeruli. Renal tubules were lined with typical cuboidal epithelium and had distinct lumen. Chronic pyelitis was observed in 1 female rat in normal control and LPE $1000 \mathrm{mg} / \mathrm{kg}$ groups (Plates 3 and 4). Thus these changes were not considered to be treatment related in this study, because these microscopic changes were observed in normal control group.

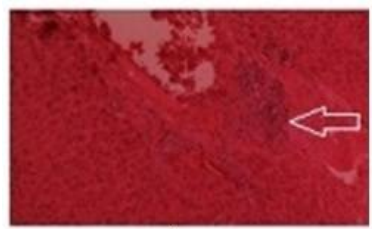

A

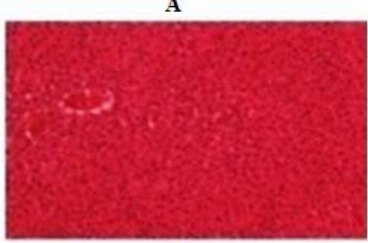

C

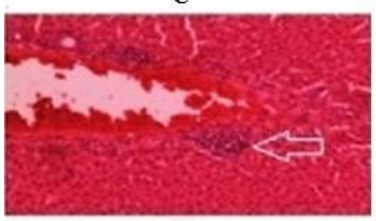

$\mathbf{E}$

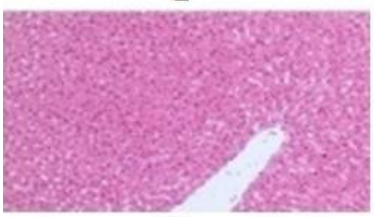

G

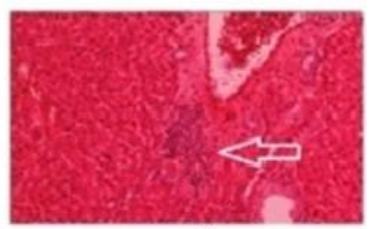

B

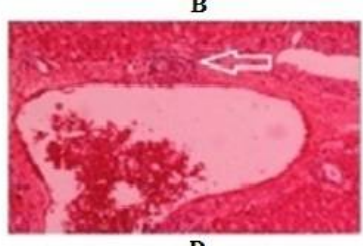

D
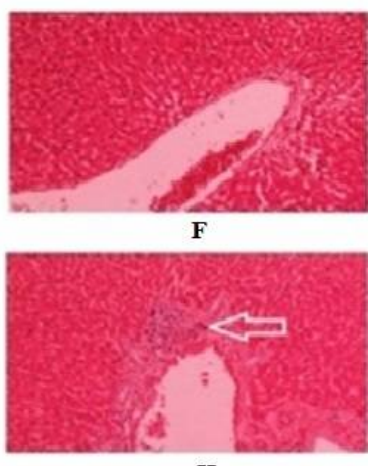

$\mathbf{H}$
Plate 1: Effects of different doses of LPE on liver histology of female and male rats in repeated dose toxicity study for 28 days as assessed by $H$ \& $E$ staining. (A): female normal control with arrow showing mild periportal chronic inflammation (1 out of 5 rats) (B): male normal control with arrow showing mild periportal chronic inflammation (only 1 out of 5 rats) (C) female treated group with $250 \mathrm{mg} / \mathrm{kg}$ of LPE (D) male treated group with $250 \mathrm{mg} / \mathrm{kg}$ of LPE with arrow showing mild periportal chronic inflammation (only 1 out of 5 rats) (E) female treated group with 500 $\mathrm{mg} / \mathrm{kg}$ of LPE with arrow showing mild periportal chronic inflammation (only 1 out of 5 rats) (F) male treated group with $500 \mathrm{mg} / \mathrm{kg}$ of LPE (G) female treated group with $1000 \mathrm{mg} / \mathrm{kg}$ of LPE $(\mathrm{H})$ male treated group with $1000 \mathrm{mg} / \mathrm{kg}$ of LPE with arrow showing mild periportal chronic inflammation (only 1 out of 5 rats) (Magnification $\times 10$ ).

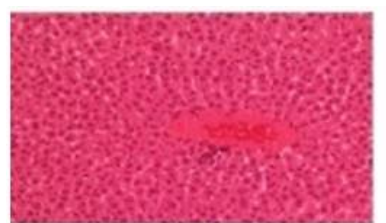

C

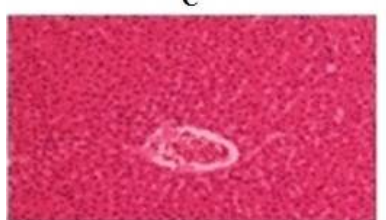

E

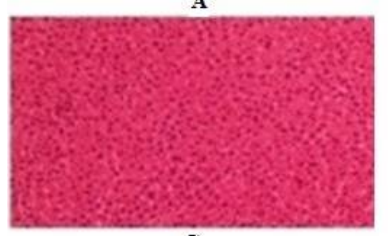

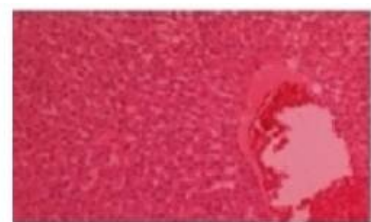

B
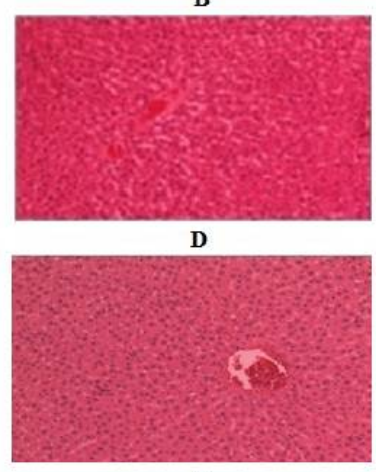

F
Plate 2: Effects of different doses of LLP on liver histology of female and male rats in repeated dose toxicity study for 28 days as assessed by $H$ \& $E$ staining. (A) female treated group with $250 \mathrm{mg} / \mathrm{kg}$ of LLP (B) male treated group with $250 \mathrm{mg} / \mathrm{kg}$ of LLP (C) female treated group with $500 \mathrm{mg} / \mathrm{kg}$ of LLP (D) male treated group with $500 \mathrm{mg} / \mathrm{kg}$ of LLP (E) female treated group with $1000 \mathrm{mg} / \mathrm{kg}$ of LLP (F) male treated group with $1000 \mathrm{mg} / \mathrm{kg}$ of LLP (Magnification $\times 10$ )

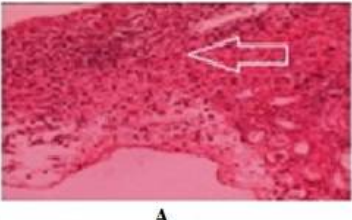

A

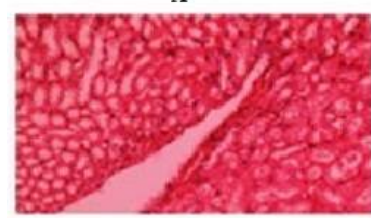

C

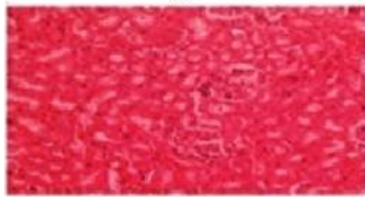

$\mathbf{E}$

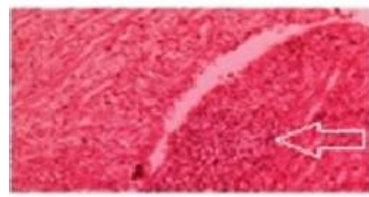

G

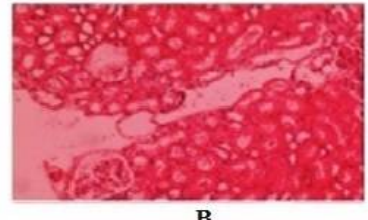

B

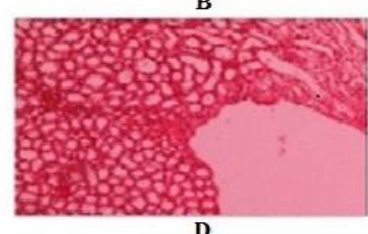

$\mathbf{F}$

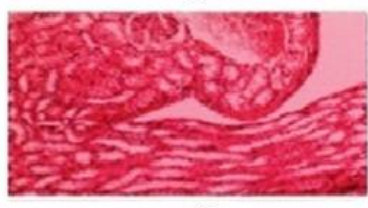

H

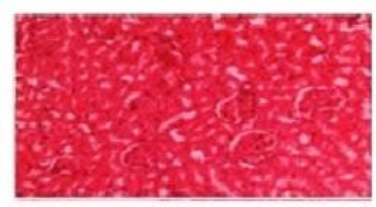

Plate 3: Effects of different doses of LPE on kidney histology of female and male rats in repeated dose toxicity study for 28 days as assessed by $H$ \& $E$ staining $(A)$ : female normal control showing normal renal tubules with chronic pyelitis (only 1 out of 5 rats) (B): male normal control group (C): female treated group with $250 \mathrm{mg} / \mathrm{kg}$ of LPE (D): male treated group with $250 \mathrm{mg} / \mathrm{kg}$ of LPE (E): female treated group with $500 \mathrm{mg} / \mathrm{kg}$ of LPE (F): male treated group with 500 $\mathrm{mg} / \mathrm{kg}$ of LPE (G): female treated group with 1000

Trop J Pharm Res, August 2018; 17(8): 1562 
$\mathrm{mg} / \mathrm{kg}$ of LPE showing normal tubules with chronic pyelitis (only 1 out of 5 rats) $(\mathrm{H})$ : male treated group with $1000 \mathrm{mg} / \mathrm{kg}$ of LPE

(Magnification $\times 10$ )
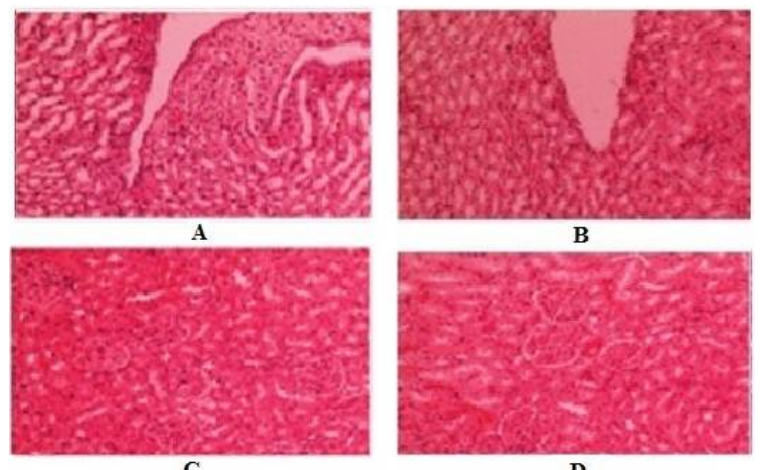

B

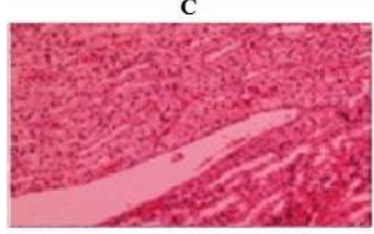

$\mathbf{E}$

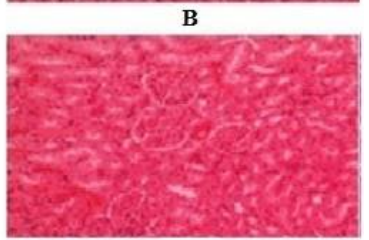

D

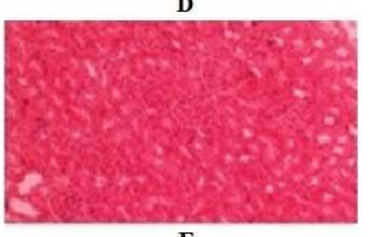

Plate 4: Effects of different doses of LLP on kidney histology of female and male rats in repeated dose toxicity study for 28 days as assessed by $\mathrm{H}$ \& $\mathrm{E}$ staining (A): female treated group with $250 \mathrm{mg} / \mathrm{kg}$ of LLP (B): male treated group with $250 \mathrm{mg} / \mathrm{kg}$ of LLP (C): female treated group with $500 \mathrm{mg} / \mathrm{kg}$ of LLP (D): male treated group with $500 \mathrm{mg} / \mathrm{kg}$ of LLP (E): female treated group with $1000 \mathrm{mg} / \mathrm{kg}$ of LLP $(\mathrm{F})$ : male treated group with $1000 \mathrm{mg} / \mathrm{kg}$ of LLP. All groups in this plate showing normal glomeruli and tubules (Magnification $\times 10$ )

\section{DISCUSSION}

Herbal medicines are gaining popularity in developing countries because of their less side effects. Such remedies are often believed to be harmless, since these treatments are "natural" and commonly used for self-medication without supervision. Although medicinal plants may cause several biological activities in humans, a little is known regarding the potential toxicity in many of these bioactive substances.

The results of acute toxicity study indicated that LPE and LLP didn't cause visible signs of toxicity or mortality. Generally, a reduction in body weight and internal organ weights is a simple and sensitive index of toxicity after exposure to potentially toxic substances [17]. In the present study, LPE and LLP (2000 mg/kg, per oral) did not significantly affect body or organ weight as compared to the normal control groups, which suggests that the extract did not disturb rat growth; this is with the agreement to the toxicity classification reported by (Loomis and Hayes 1996) [18].
However a 28-days sub-chronic oral toxicity testing using repeated doses is widely considered satisfactory to assess any possible health hazard, and is important even for herbal supplements. The present results revealed that all parameters in term of organ-to-body weight ratios, haematological biochemical values were found normal between LPE and LLP treated groups compared to normal control group [16]. Histological examination of the vital organs including the liver and kidney from the treated and normal rats showed no apparent histological changes, chronic pyelitis (inflammation of renal pelvis and calyces), was only focally seen at pelvis of the kidney. It is often caused by ascending bacterial infection. The renal parenchyma was normal in this study. Overall the histological examination indicated evidence of safety at the tested doses when administered orally. The safety of treatment was also confirmed by the absence of behavioural changes and absence of difference in feed consumption between the treated and normal control groups. Hence, the results clearly showed that oral administration of LPE and LLP are safe at the evaluated doses (250, 500 and 1000 $\mathrm{mg} / \mathrm{Kg}$ ) in Sprague Dawley rats.

\section{CONCLUSION}

The acute and repeated dose oral toxicity assay of LPE and LLP could be very useful in its future clinical studies. LPE and LLP seemed to be nontoxic as was seen after its acute and repeated dose oral administrations. The oral lethal dose is in excess of $1000 \mathrm{mg} / \mathrm{kg}$. The no-adverse-effectlevel from the present study was determined to be $1000 \mathrm{mg} / \mathrm{kg}$ per day for rats under the condition tested.

\section{DECLARATIONS}

\section{Acknowledgement}

The authors wish to thank Ministry of Agriculture, Malaysia for providing the financial support, grant no. 304/PFARMASI/650603/K123, and also Universiti Sains Malaysia for the lab facilities.

\section{Conflict of interest}

No conflict of interest associated with this work.

\section{Contribution of authors}

The authors declare that this work was done by the authors named in this article and all liabilities pertaining to claims relating to the content of this article will be borne by them. Guarantors of 
integrity of entire study, Zhari Ismail, Amirin Sadikun, Mohammed Ali Ahmed Saeed and Abdul Hakeem Memon; study concepts/study design or data acquisition or data analysis/interpretation, all authors; manuscript drafting or manuscript revision for important intellectual content, all authors; manuscript final version approval, all authors; literature research, Mohammed Ali Ahmed Saeed and Sultan Ayesh Mohammed Saghir; experimental studies, Mohammed Ali Ahmed Saeed, Abdul Hakeem Memon, Mohd Shahrul Ridzuan Hamil and Sultan Ayesh Mohammed Saghir; histology assay, Gurjeet Kaur; statistical analysis, Mohammed Ali Ahmed Saeed and Hooi Kheng Beh; and manuscript editing, Zhari Ismail, Amirin Sadikun and Abdul Hakeem Memon.

\section{REFERENCES}

1. Karimi E, Jaafar HZ, Ahmad S. Phytochemical analysis and antimicrobial activities of methanolic extracts of leaf, stem and root from different varieties of Labisia pumila Benth. Molecules 2011; 16 (6): 4438-4450.

2. Dianita R, Jantan I, Amran AZ, Jalil J. Protective effects of Labisia pumila var. alata on biochemical and histopathological alterations of cardiac muscle cells in isoproterenol-induced myocardial infarction rats. Molecules 2015; 20 (3): 4746-4763.

3. Rice-Evans CA, Miller NJ, Paganga G. Structureantioxidant activity relationships of flavonoids and phenolic acids. Free Radic Biol Med 1996; 20 (7): 933956.

4. Fiuza SM, Gomes C, Teixeira LJ, Da Cruz MG, Cordeiro MN, Milhazes N, Borges F, Marques MP. Phenolic acid derivatives with potential anticancer properties-a structure-activity relationship study. Part 1: Methyl, propyl and octyl esters of caffeic and gallic acids. Bioorg Med Chem 2004; 12 (13): 3581-3589.

5. Al-dhabi NA, Arasu MV, Park CH, Park SU. Letter to the editor: An up-to-date review of rutin and its biological and pharmacological activities. Excli J 2015; 14, 59-63.

6. Choubey S, Varughese LR, Kumar V, Beniwal $V$. Medicinal importance of gallic acid and its ester derivatives: a patent review. Pharm Pat Anal 2015; 4 (4): 305-315.

7. Subramanian $A P$, John $A A$, Vellayappan $M V$, Balaji $A$, Jaganathan SK, Supriyanto E, Yusof M. Gallic acid: prospects and molecular mechanisms of its anticancer activity. RSC Adv 2015; 5 (45): 35608-35621.

8. He Z, Chen AY, Rojanasakul Y, Rankin GO, Chen YC. Gallic acid, a phenolic compound, exerts anti-angiogenic effects via the PTEN/AKT/HIF-1a/VEGF signaling pathway in ovarian cancer cells. OncolRep 2016; 35 (1): 291-297.

9. Singh GD, Ganjoo M, Youssouf MS, Koul A, Sharma R, Singh S, Sangwan PL, Koul S, Ahamad DB, Johri RK. Sub-acute toxicity evaluation of an aqueous extract of Labisia pumila, a Malaysian herb. Food Chem Toxicol 2009; 47 (10): 2661-2665.

10. Wan EMF, Amrah S, Mohamad NI, Mohd SAW, Syed MSJ. Evaluation of the Teratogenicity of Aqueous Extract of Labisia pumila var. alata in Rats. Malays $J$ Med Sci 2005; 12: 13-21.

11. Ezumi MW, Amrah SS, Suhaimi AW, Mohsin SS. Evaluation of the female reproductive toxicity of aqueous extract of Labisia pumila var. alata in rats. Indian J Pharmacol 2007; 39 (1): 30.

12. Aisha AF, Majid AM, Ismail Z. Preparation and characterization of nano liposomes of Orthosiphon stamineus ethanolic extract in soybean phospholipids. BMC Biotechnol 2014; 14 (1): 1-11.

13. OECD guidelines for the testing of chemicals acute oral toxicity-Up-and-Down-Procedure (UDP), October 2008.

14. Ghosh M. Fundamentals of experimental pharmacology. Indian J Pharmacol 2007; 39 (4): 216-221.

15. OECD guidelines for the testing of chemicals, Repeated Dose 28-Day Oral Toxicity Study in Rodents, October 2008.

16. Lillie $L E$, Temple NJ, Florence $L Z$. Reference values for young normal Sprague-Dawley rats: weight gain, hematology and clinical chemistry. Hum Exp Toxicol 1996; 15 (8): 612-616.

17. Teo $S$, Stirling $D$, Thomas $S$, Hoberman A, Kiorpes $A$, Khetani V. A 90-day oral gavage toxicity study of $d$ methylphenidate and d, I-methylphenidate in SpragueDawley rats. Toxicol 2002; 179 (3): 183-196.

18. Loomis TA, Hayes AW. Loomis's essentials of toxicology. Academic press; 1996. 